九州大学学術情報リポジトリ

Kyushu University Institutional Repository

Programming for Time-frequency Analys is of Transient Seismic Signals by Multiple Filter Technique and Running Spectrum Technique and Its Application

Wang, Yanbin

Faculty of Sciences, Kyushu Univeristy

Takenaka, Hiroshi

Faculty of Sciences, Kyushu University

https://doi. org/10.5109/1546560

出版情報: 九州大学大学院理学研究院紀要：Series D, Earth and planetary sciences. 30 (1)， pp. 45-57, 1998-01-30. Faculty of Science, Kyushu University

バージョン :

権利関係 : 
Mem. Fac. Sci., Kyushu Univ., Ser. D, Earth Planet. Sci., Vol. XXX, No. 1, pp. 45-57, text-figs. 1-7, January 30, 1998

\title{
Programming for Time-frequency Analysis of Transient Seismic Signals by Multiple Filter Technique and Running Spectrum Technique and Its Application
}

\author{
Yanbin WANG and Hiroshi TakenaKa
}

\begin{abstract}
Time-frequency analysis is one of useful signal processing tools for seismic signals. Several techniques for time-frequency analysis have been developed for different applications. In this study, the multiple filter technique and the running spectrum techniques based on maximum entropy method (MEM) and Fourier transform have been employed. Three programs for time-frequency analysis by multiple filter technique, MEM and Fourier transform, respectively, have been coded in FORTRAN. The property, especially time and frequency resolutions of the three methods were compared between each other through case studies. Results show that which method should be used in an application depends on the purpose of research and the characteristics of the time signals. As a critical factor for understanding and explaining the results, graphic visualization of the outputted data has also been studied.
\end{abstract}

\section{Introduction}

Seismic signal contains information from the source which generates the signal, the medium in which it propagates and the instrument which records the signal. In order to extract useful information for particular purpose, data processing techniques are manipulated for seismic signal. Generally, the time series is described in different ways after data processing which has numerous advantages over direct readings from time series for understanding. As one of the commonly adopted techniques, joint time-frequency domain analysis treats two fundamental variables, time and frequency, represents the signal in terms of temporal variation of frequency component. Spectral decomposition in 2-D time-frequency domain reveals much more information than the 1-D time domain and 1-D frequency domain spectrum representations. In seismology, joint time-frequency analysis has been used in the following applications.

Dispersed seismic signal varies simultaneous with time and frequency. Joint timefrequency analysis has been extensively used to get dispersion character of surface wave and calculate dispersion curve of it for natural earthquake, and the result was used to inverse the shallow structure of the Earth (e.g., DOO and RICHARD, 1991 ; CHUNG and YEONG, 1997).

For seismic signal generated by earthquake of great magnitude and propagating through long distance, and with sophisticated local site geology, it is not so easy to identify different phases and separate wave types directly from the complicated seismograms. When the amplitude or energy variation of the signal is presented both in the time and frequency

Manuscript received September 26, 1997 ; accepted October 24, 1997. 
domain, the phases and wave types can be clearly identified and separated which can be used to interpret wave propagation effect (e.g., YOKOI, 1996).

Time-frequency analysis has also been used to study the frequency component of seismic signal and infer the effect of propagation medium and local site geology. For example, comparing the time-frequency representations for seismograms recorded by the same station for main shock and related aftershocks, for those recorded by different stations for the same earthquake, the local site effect can be detected (e. g., FurUmuRA, 1994).

There are several time-frequency analysis techniques applied not only in seismology, but also in other fields (KOBAYASHI and SAKAMOTO, 1997). Such as techniques based on Fourier transform, wavelet transfrom, maximum entropy method and Wigner distribution. Previous application of these techniques show that appropriate method should be used for one special study.

Information extracted from strong motion data is very valuable for seismic disaster reduction research. Strong motion data generally contains wide frequency range. In order to analyse this kind of data, the multiple filter technique based on Fourier transform and running spectrum technique based on Fourier transform and maximum entropy method have been applied. Programs for the three methods have been coded referred to the previous works. Time frequency resolution and their applicability in strong motion data analysis have been compared through case studies. How to display the result data is important for understanding and explaining them. Good display can reveal more and clear information concealed in the result. How to perform the data visualization is also studied in the case studies.

\section{Methods, algorithms and programs}

\subsection{Multiple Filter Technique}

Multiple filter technique is an algorithm developed by DZIEWONSKI, BLOCH and LANDISMAN (1969) aimed to compute the dispersion curves of seismic surface waves. It is used to study variations of amplitude (or energy) of a signal as a function of time (velocity) and frequency (period). This analytical method was shown to be a fast and efficient means of studying multi-mode dispersed seismic signal. Comparing with the classical peak and trough method, it can recover broader portions of the dispersion present in ordinary seismic recordings (DZIEWONSKI et al., 1969). It has been widely used in studying seismic surface waves and the frequency component of seismic signal.

By this technique, a seismic signal, $f(t)$, is first Fourier transformed into $F(\omega)$. Then filtered in the frequency domain using a Gaussian function represented by

$$
G(\omega)=\exp \left[-\alpha\left(\frac{\omega-\omega_{n}}{\omega_{n}}\right)^{2}\right]
$$

where $\omega$ is the angular frequency, $\omega_{n}$ is the center frequency, and

$$
\alpha=\beta / B^{2}
$$

The parameter $\beta$ describes the decay of the window function, which is determined by the desired value of the function at the band limits. $B$ is the relative half-bandwidth.

The filtered seismic signal $F_{n}(\omega)$ for each $\omega_{n}$ is inverse Fourier transformed and its 


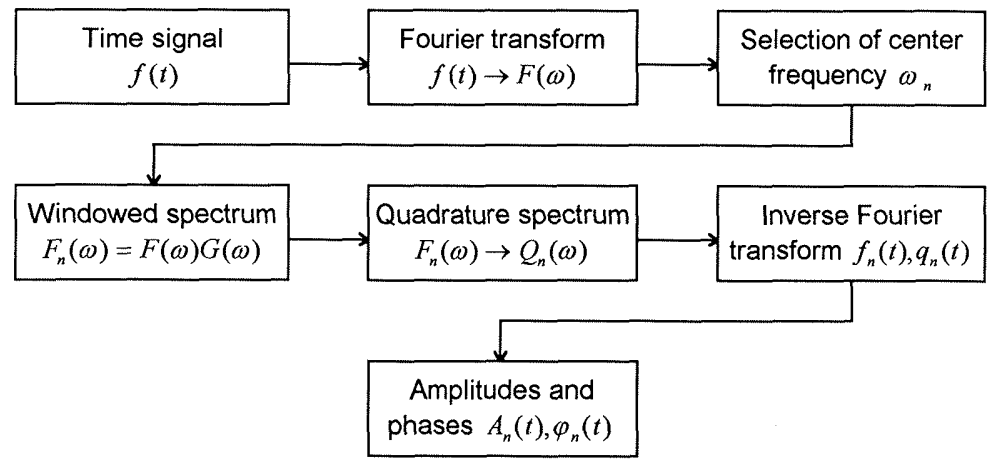

Fig. 1. Flow-chart of the multiple filter analysis process.

output is designated by $f_{n}(t)$. The Hilbert transform of $f_{n}(t)$ gives the quadrature signal of it as $q_{n}(t)\left(Q_{n}(\omega)\right.$ in the frequency domain). The instantaneous spectral amplitudes $A_{n}(t)$ and phases $\varphi_{n}(t)$, respectively, are defined as (GOODMAN, 1960)

$$
A_{n}(t) \cdot e^{i \varphi_{n}(t)}=f_{n}(t)+i q_{n}(t)
$$

When the instantaneous amplitudes $A_{n}(t)$ is plotted as a function of frequency and time (or period and group velocity), the temporal variation of frequency component and the dispersion relationships of seismic signal $f(t)$ can be identified.

Based on the algorithm described above, a program was coded in QuickBASIC by DoO and RICHARD (1991). Their program is originally aimed to calculate the dispersion curve of surface waves generated in exploration seismic surveys. Referring to their code, we made a FORTRAN program based on the same algorithm. In this program, a time signal is first Fourier-transformed into the frequency domain after pre-processing, and then filtered by a series of frequency domain Gaussian filters of different center frequency. The filtered signal in the frequency domain is inverse Fourier transformed into the time domain, the analytical time-domain signal is obtained by Hilbert transform. The instantaneous amplitude is finally outputted, smoothed by running-mean process and displayed in the time-frequency space. Figure 1 gives the flow-chart of our code.

\subsection{Running Spectra Technique}

The running spectrum technique is to perform a spectral analysis for a portion of a time series by moving time windows. A selected time window $w(t)\left(t \in\left[t_{1}, t_{2}\right]\right)$ is applied to the time series $f(t)(t \in[0, \infty))$, the spectrum is then estimated for the small section of the windowed signal $x(t)=f(t) w(t)\left(t \in\left[t_{1}, t_{2}\right]\right)$. By shifting this time window appropriately over the total time series, the frequency content of the signal is extracted and a $2-D$ representation of frequencies versus time is produced. Here, we apply the running spectrum technique based on the maximum entropy method or Fourier transform for estimating power spectrum for time series.

\section{Maximum Entropy Method (MEM)}

The MEM method is proposed by BURG in 1967 for improving the resolution for power 
spectrum estimation (HINO, 1977). For a time series of normal distribution, its entropy $H$ is proportional to the following integration

$$
H \propto \int_{-f_{N}}^{f_{N}} \log P(f) d f
$$

where $P(f)$ is the power spectrum of the time series, $f_{N}$ is the Nyquist frequency. The power spectrum $P(f)$ and the auto-correlation function $C_{k}$ of the time series are connected by the Wiener-Khintchine's equation

$$
\int_{-f_{N}}^{f_{N}} P(f) z^{k} d f=C_{k}
$$

where

$$
z=\exp \{i 2 \pi f \Delta t\}
$$

The MEM method assures the entropy of the time series to be the maximum when the unknown auto-correlation are inferred for power spectrum estimation. For keeping the entropy to be the maximum, estimation of power spectrum can be written as

$$
P(f)=\frac{P_{m} \Delta t}{\left|1+\sum_{k=1}^{m} \gamma_{k} e^{i 2 \pi f b d t}\right|^{2}}
$$

where $\gamma_{k}$ is the $m$ point prediction error filter, and $P_{m}$ is the average output from this filter.

We made a FORTRAN program to carry out the running spectrum technique using MEM. A time signal is firstly pre-processed by removing mean. An appropriate time window function is applied to the time signal, power spectrum for each windowed signal is calculated by moving this window in a constant time lag. The outputted power spectrum is then smoothed in the time domain by running mean process and finally displayed in the timefrequency space. We have employed an efficient subroutine for estimating power spectrum by MEM (HINO, 1977). In this subroutine, the Burg method is used to solve the Yule-Walker equations for calculating the coefficients $\gamma_{k}$ of prediction error filter and output of this filter $P_{m}$. The flow-chart of this program is shown in Figure 2 .

\section{Fourier Method}

Fourier transform can also be used for power spectrum estimation. A time function $f(t)$

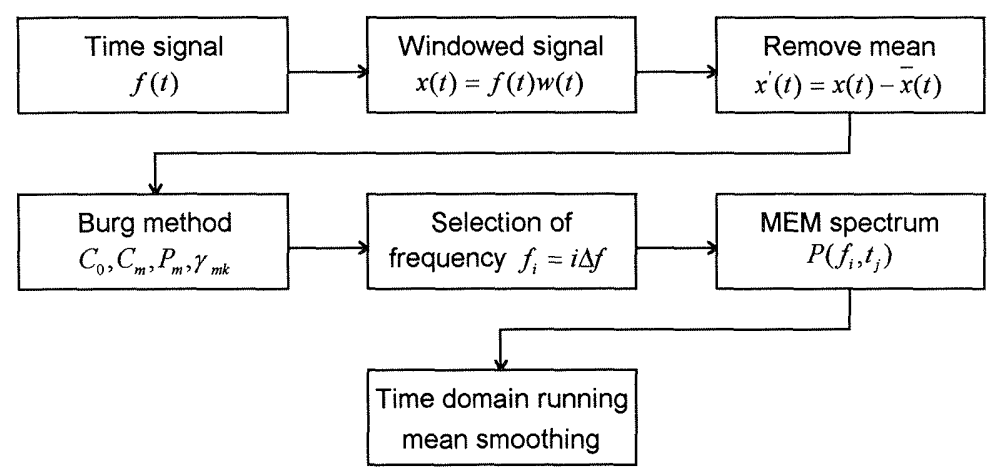

Fig. 2. Flow-chart of the running spectrum analysis process by MEM. 


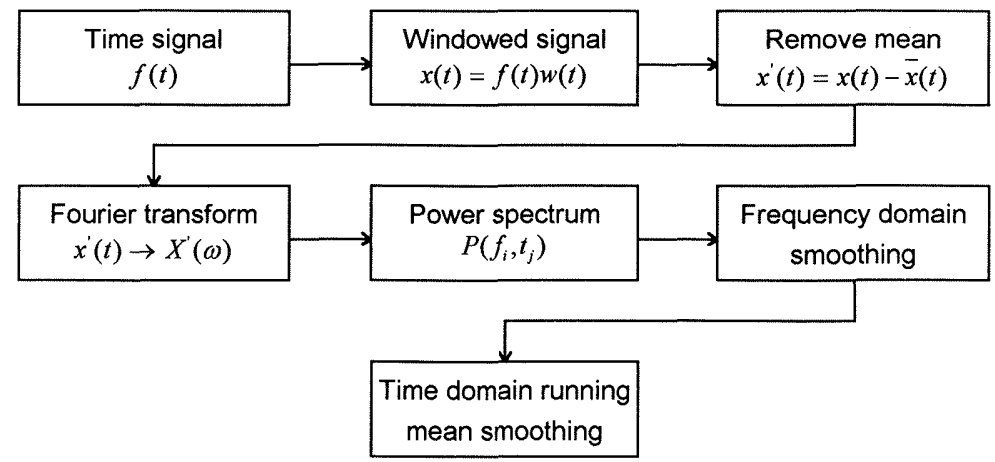

Fig. 3. Flow-chart of the running spectrum analysis process by Fourier transform.

is Fourier transfromed into the frequency domain by

$$
F(f)=\int_{-\infty}^{\infty} f(t) e^{-i 2 \pi f t} d t
$$

In this study, the power spectrum is calculated and displayed in the result. Since the power spectrum can be directly evaluated from the Fourier amplitude spectrum calculated by Fourier transform because the relationship between auto-correlation and Fourier amplitude spectrum (HiNO, 1977).

We also constructed a FORTRAN program that includes a FFT subroutine of HINO (1977). First, the time window is applied to a time signal. The windowed signal is preprocessed by removing mean, and Fourier-transformed by the FFT. Power spectrum is then calculated and smoothed in the frequency domain by a Bartlett filter. The smoothed power spectrum is outputted for the time frequency matrix. Finally, the power spectrum is also smoothed by running mean process in the time domain. The flow-chart of this program is given in Figure 3.

\subsection{Resolutions in joint time-frequency analysis}

For spectrum estimation, the frequency resolution is vital for separating frequency components of time signal. For joint time-frequency analysis, except for the frequency resolution, the time resolution is also very important for locating the frequency content. Unfortunately, good resolutions in both time and frequency domains can not be achieved simultaneously, the improvement of resolution in one domain is achieved at the expenses of the resolution in the other domain. This is supported by the uncertainty principle.

Numbers $d$ and $D$ are defined as the measures of the time-domain duration of $f(t)$ and the related frequency-domain duration of $F(\omega)$

$$
\begin{aligned}
& d^{2}=\frac{1}{E} \int_{-\infty}^{\infty} t^{2}|f(t)|^{2} d t \\
& D^{2}=\frac{1}{2 \pi E} \int_{-\infty}^{\infty} \omega^{2}|F(\omega)|^{2} d \omega
\end{aligned}
$$

Then the frequency-time resolution can be measured as $1 / D d$ which is hold for the following equality and inequality (PAPOULIS, 1977) 


$$
D d \geq \frac{1}{2}
$$

where the equality holds only for the Gaussian function. This means the $D d$ value for the Gaussian function is constant and minimum.

For the multiple filter technique, as the signal is filtered in the frequency domain by using a Gaussian function, it gives the best combined time and frequency resolution according to the above equality. But the resolution is not the same over the analyzed frequency and time domain. Because relative bandwidth $B$ is the controlling parameter and is constant in one calculation, the absolute bandwidth increases with the increasing of the center frequency. For low frequency range, the filtered signal contains less frequency component than those for high frequency range. The frequency resolution is good in low frequency range, while the time resolution is good in high frequency range. Increasing of $B$ value will enlarge the bandwidth of Gaussian function, so it will improve the time resolution and decrease the frequency resolution.

A synthetic time signal is produced to check the time and frequency resolution of our programs described in the preceding subsections $(2.1,2.2)$. The signal is synthesized by adding three sine waves of frequencies 10,20 and $30 \mathrm{~Hz}$. The sampling frequency is $100 \mathrm{~Hz}$. Two spikes are presented at approximately 2.4 and 2.7 seconds. Figure 4 presents the timefrequency analysis results for this signal by multiple filter technique. In this analysis, 80 frequency filters have been used between 0.5 to $40 \mathrm{~Hz}$. The $B$ value is $0.025,0.1,0.2$ and 0.3 , respectively, for Figure 4(a) to Figure 4(d). The data displayed in Figure 4 is instantaneous amplitude in $\mathrm{dB}$ which is normalized so that the maximum value is 100 . The dark color represents the large instantaneous amplitude. Details for display technique will be explained in section 3. In Figure 4(a), the three sine waves are well resolved, but the individual spikes are not separated and presented as average. In Figures 4(c) and 4(d), the two spikes appear clearly, but the sine waves of 20 and $30 \mathrm{~Hz}$ are mixed together. Figure 4(b) presents both good frequency and time resolution, the three sine waves and two spikes are separated. Small $B$ value produces good frequency resolution, and large $B$ value produces good time resolution. In all the four figures, the high frequency range has good time resolution and the low frequency range has good frequency resolution. The $B$ value should be carefully selected according to the purpose of analysis.

Figure 5 shows the analysis results for the same synthetic signal by the running spectrum method which is displayed in the same way as Figure 4. Figures 5(a), 5(c) and 5(e) are the results by Fourier transform of time window length $0.07,0.63$ and 2.55 seconds which corresponds to $2^{3}, 2^{6}$ and $2^{8}$ data, respectively. Figures 5(b), 5(d) and 5(f) are by MEM with the same window length. In both cases, the two spikes can be well located as shown in Figures 5(a) and 5(b) for short time window as the window length is smaller than the spacing between the spikes. But the three sinusoids are averaged together and can not be identified. Figures 5(e) and 5(f) shows the three well localized sinusoids for long time window, but the two spikes are averaged over wide range of frequency. Figures 5(c) and 5(d) show better location of the three sine waves and the two spikes compare with other results. For running spectrum technique by both Fourier transform and MEM, the frequency resolution improves with the increasing of time window length, while the time resolution improves with the decreasing of window length. To get a better presentation in both time and frequency domain, it is important to choose the appropriate length for time window. 
Figure 6 shows the running spectrum results of the same frequency resolution by Fourier transform and MEM which is also displayed as in Figures 4 and 5. Figure 6(a) is calculated by Fourier transform with time window of 2.55 seconds. Figure $6(\mathrm{~b})$ is the result by MEM with time window of 1.08 seconds. The MEM achieves the same frequency resolution with short time window. The two spikes are better located in Figure 6(b) than in Figure 6(a), the time domain resolution for MEM is better than Fourier transform for the same frequency resolution. For the analysis by Fourier transform, selection of time window is restricted, and the frequency resolution is directly related to the time window length as the Fourier transform maps the time series to the same number of frequency series in the frequency domain. But for MEM, the time window can be selected in arbitrary length, the frequency resolution is not directly related to the window length. The frequency interval can also be selected to
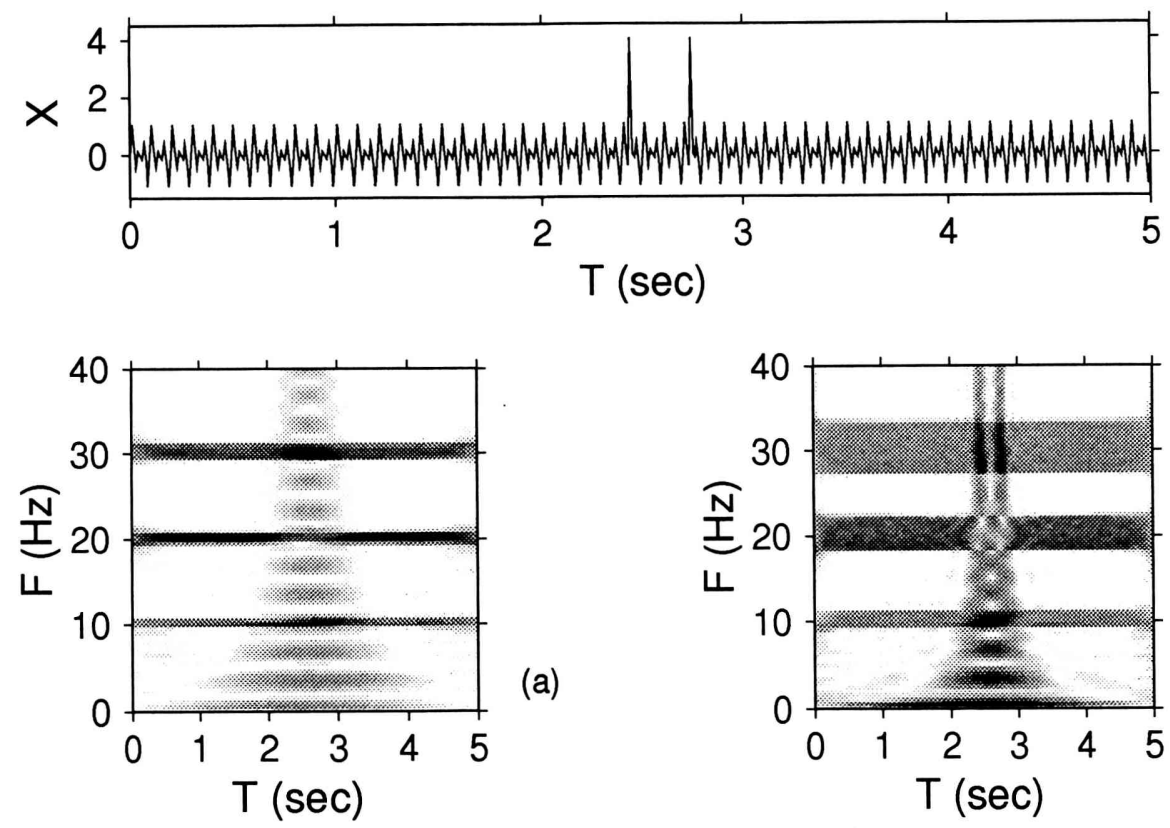

(a)

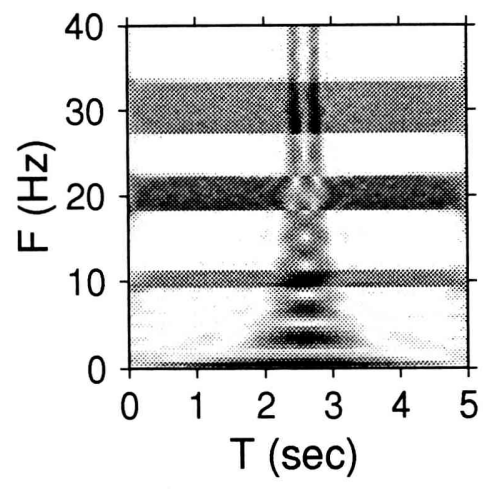

(b)
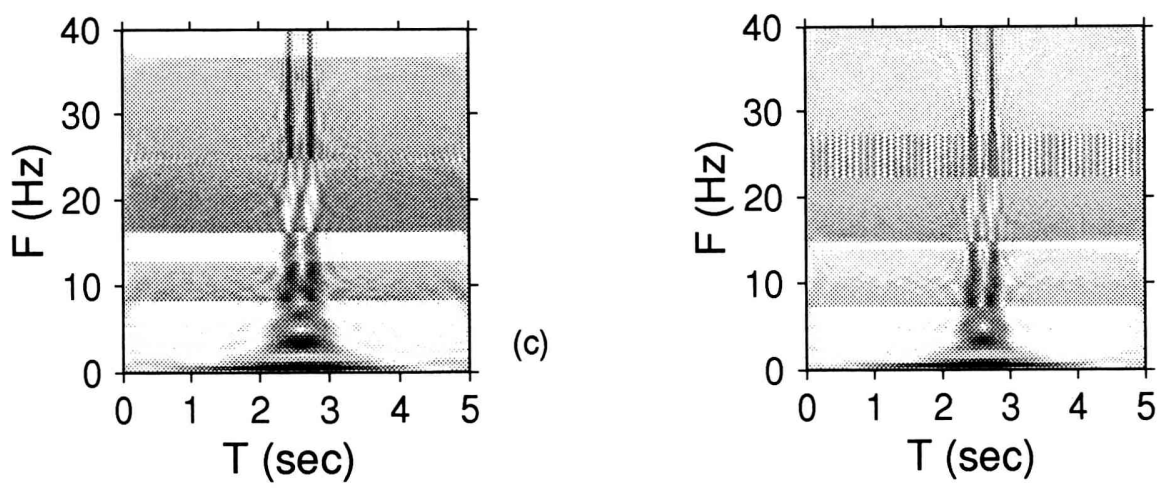

(d)

Fig. 4. The multiple filter analysis for a synthetic time signal. The top shows the synthetic time signal. Relative bandwidth is $0.025,0.10,0.20$, and 0.30 , respectively, for (a) (b) (c) and (d). 

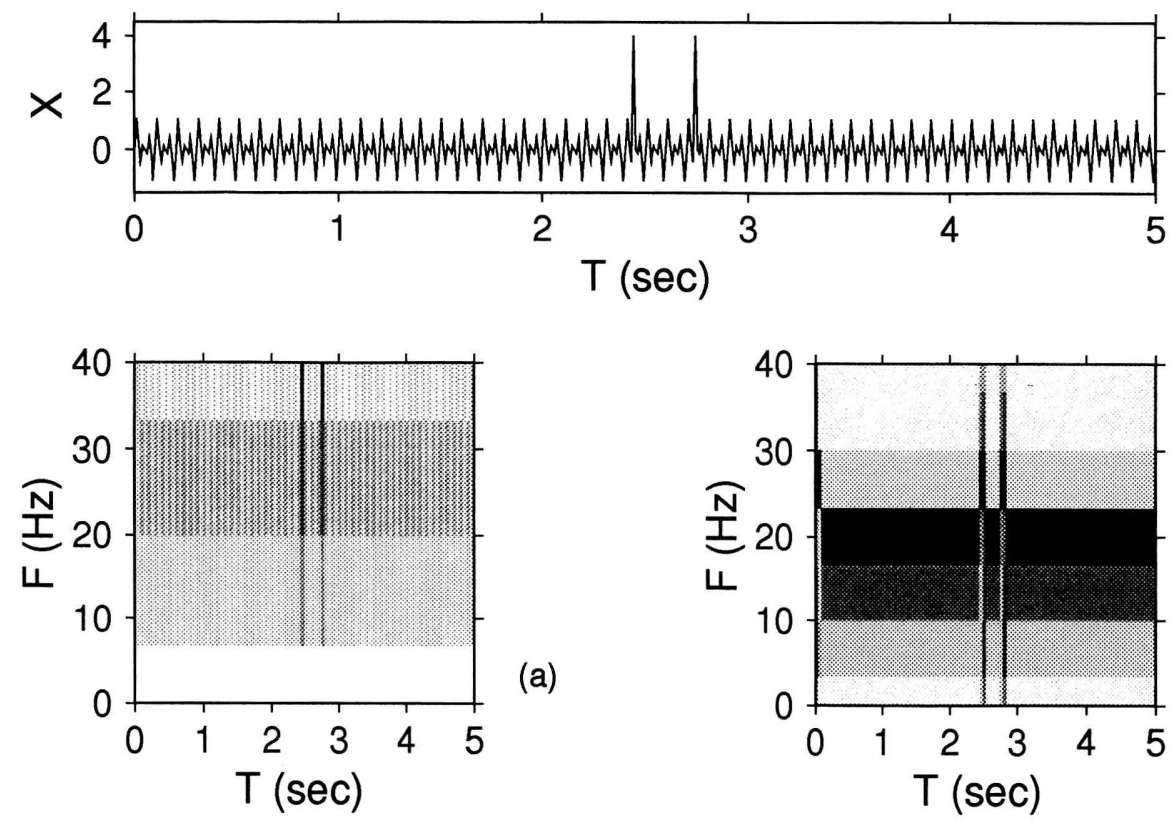

(a)

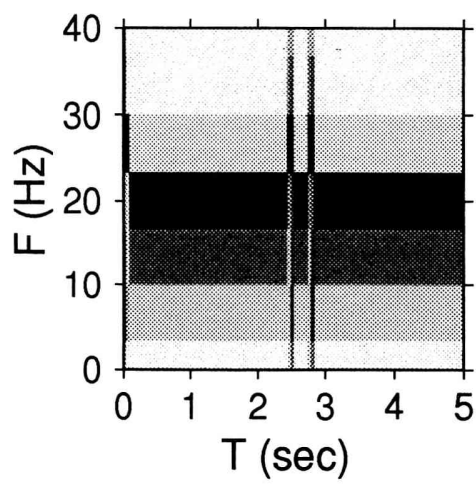

(b)
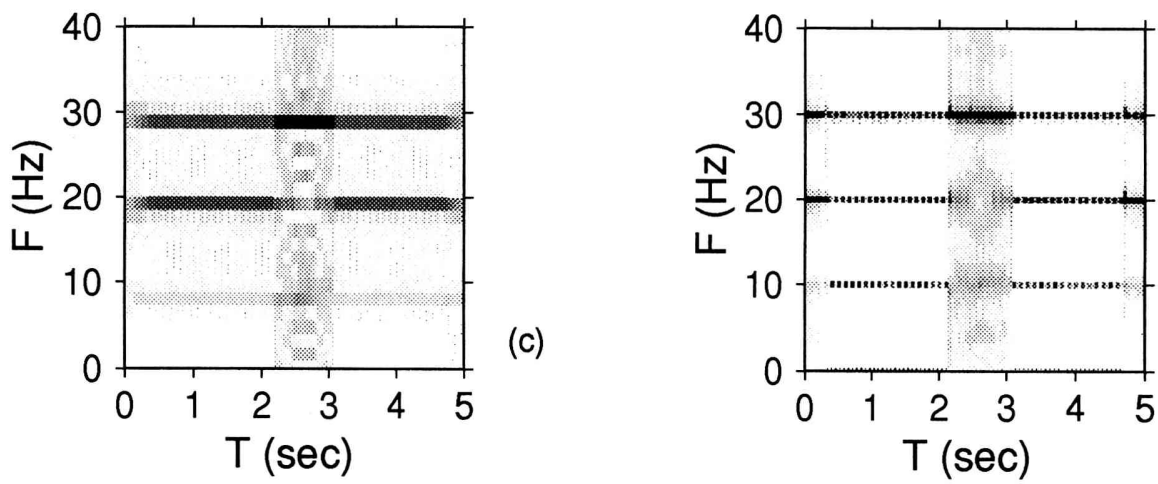

(d)
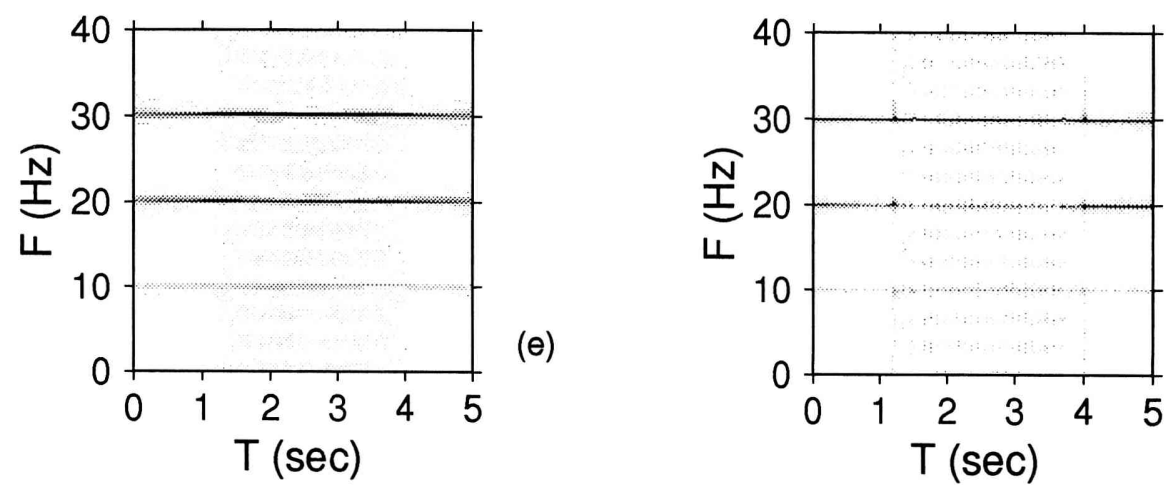

(f)

Fig. 5. Running spectrum analysis by MEM and Fourier transform. The top shows the synthetic time signal. (a) (c) and (e) are the results by Fourier transform with time window of $0.07,0.63$ and 2.55 seconds. (b) (d) and (f) are the results by MEM with the same time window as (a) (c) and (e). 

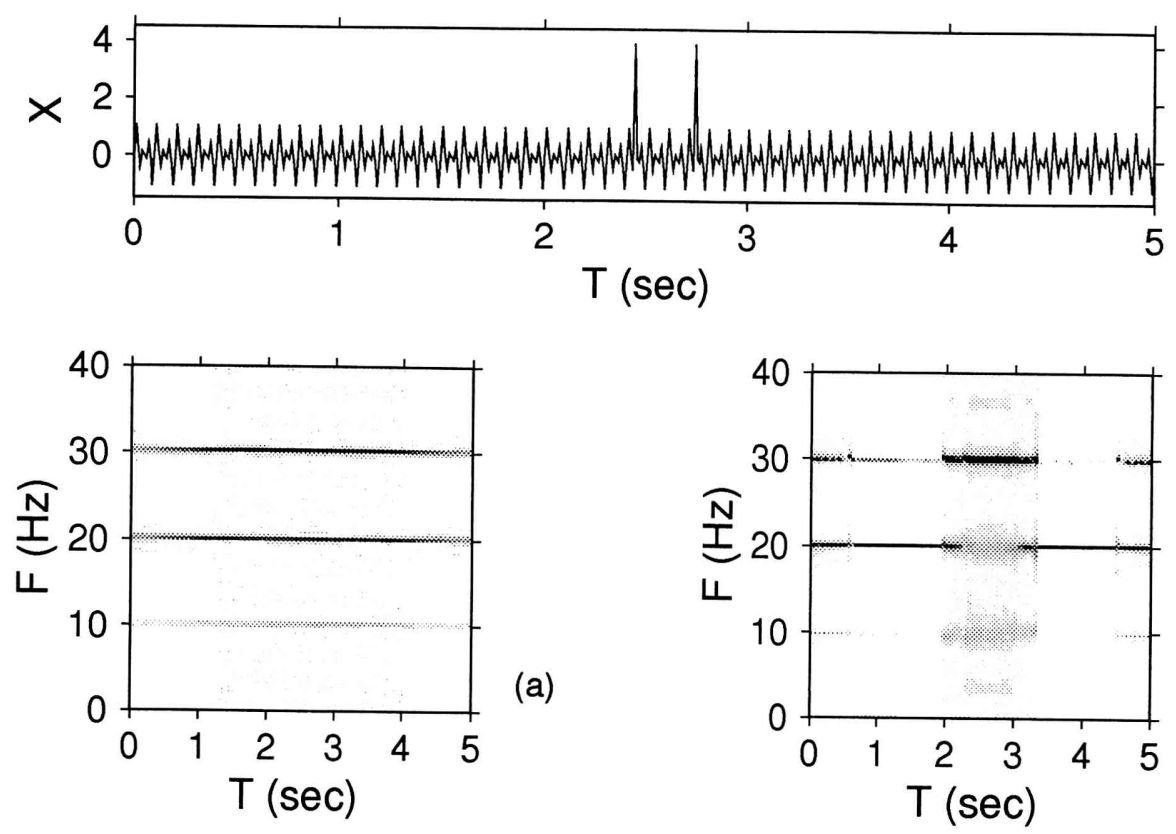

Fig. 6. Running spectrum analysis by Fourier transform and MEM. The top shows the synthetic time signal. (a) Fourier transform with time window of 2.55 seconds. (b) MEM result which has the same frequency resolution as (a), but a time window of 1.08 seconds.

certain extent.

\section{Case study and display technique}

For the purpose of testing and comparing these methods, one accelerogram recorded by the Kyoshin Net for an earthquake of magnitude 6.2 occurring on May 13, 1997 in Kagoshima was analyzed. For that generated by strong earthquake, the accelerogram contains wide range of frequency component and complicated phases and wave types. The time-frequency analysis technique which gives good resolution in wide frequency range and long time duration is desired.

The vertical component of accelerogram observed at KGS006 station at Yokokawa is displayed in Figure 7. Maximum acceleration is $95.0 \mathrm{gal}$ and sampling frequency is $100 \mathrm{~Hz}$. Look at the accelerogram, we observe that the early section of it is mainly composed of high frequency component up to about 6.0 seconds, wide frequency range between 6.0 and 12.0 seconds, very low frequency component after 12.0 seconds.

Figure 7 shows the results analyzed by the three methods. Data visualization technique is important for understanding and explanation the information contained in the result. How to display the results in time-frequency plane determines how much information the illustration contains. The UNIX based software, the Generic Mapping Tools (GMT), is used in this study for graphic display. In order to illustrate the output data clearly, two kinds of display are used here. For multiple filter analysis, the values of calculated results are very small and change in a wide range. For better display, the instantaneous amplitude is outputted in decibels $(\mathrm{dB})$ and normalized so that their greatest value is 100 . The $\mathrm{dB}$.values is calculated 
by

$$
I A=10 \log _{10}\left(\frac{A}{A_{\max }}\right)^{2}
$$

where $I A$ is the values in $\mathrm{dB}, A$ and $A_{\max }$ are the instantaneous amplitude and its maximum value normalized by $A_{\max }$. The values change abruptly from grid to grid, so it is better to display them in monochromatic picture. The number of the amplitude matrix is $2048 \times 101$, it is possible to get a monochromatic display of good continuity. With contoured map, the
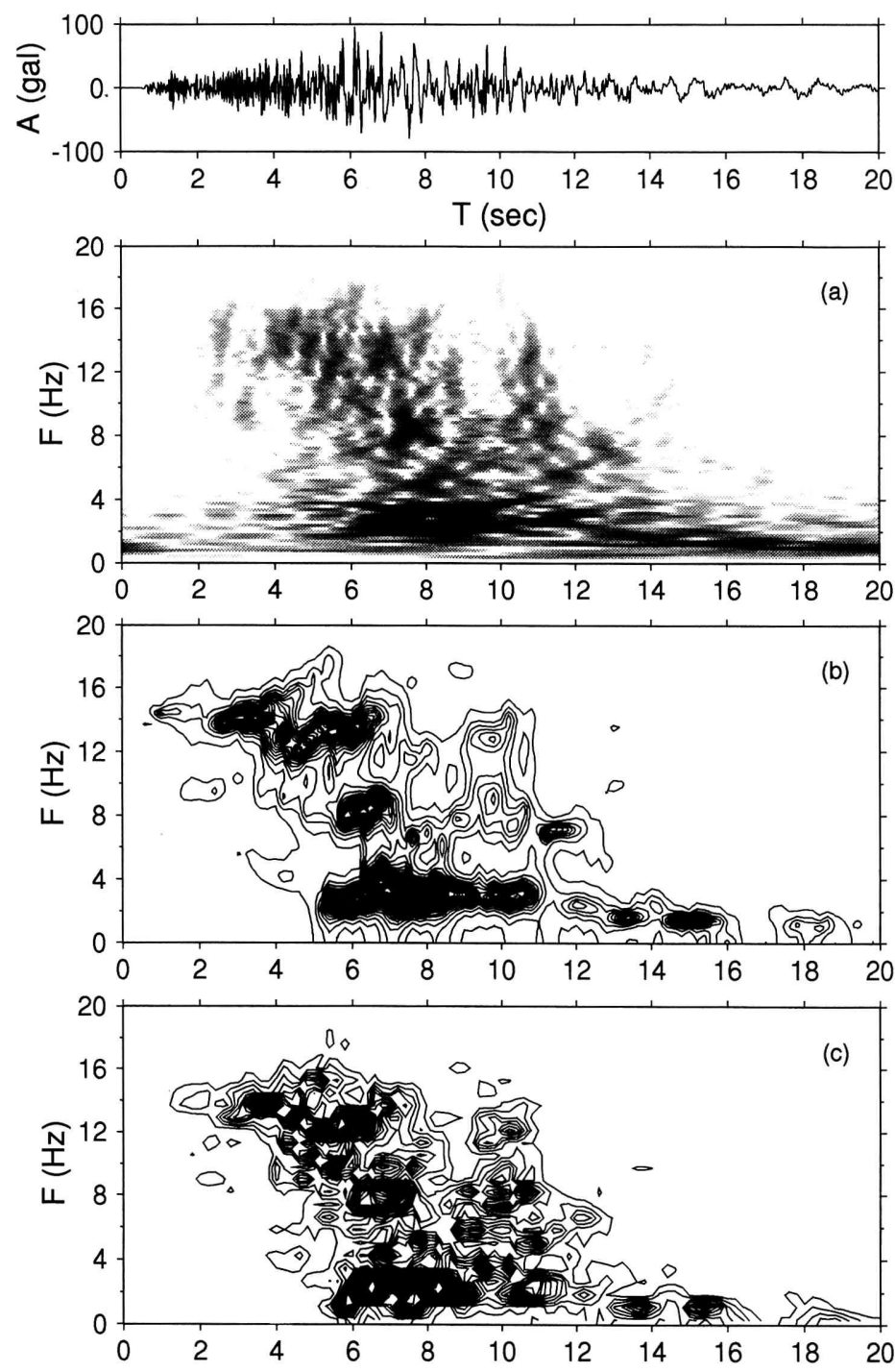

Fig. 7. Analysis results for one accelerogram of the May 13, 1997 Kagoshima earthquake of magnitude 6.2. (a) Result of multiple filter analysis. (b) Running spectrum by MEM. (c) Running spectrum by Fourier transform. 
detailed variation is proved to be merged. The gradation used in the display varies linearly with the increasing of the amplitude $\mathrm{dB}$ values. The cutoff value for display is $80 \mathrm{~dB}$. For running spectrum analysis by MEM and Fourier transform, the output data is power spectrum and the data matrix are $101 \times 128$ (the frequency steps is selected as 128 between 0 and $20 \mathrm{~Hz}$ ) and $101 \times 64$ (the frequency steps can be calculated within the Nyquist frequency for a time window of 1.28 seconds is 64 ), respectively. The values of data varies in a smaller range than those from multiple filter analysis result. The contoured map is tested to be good for running spectrum display. The results by MEM and FFT were shown in contoured map with contour interval 4.0 and 5.0.

First, we check the results of the multiple filter technique. We calculated the cases for $B$ of $0.05,0.1,0.2$ and 0.3 . The result of $B=0.1$ illustrated in Figure 7(a) gives the appropriate time and frequency resolution to identify the temporal frequency variation for all the accelerogram. Frequency range for analysis is from 0.2 to $20 \mathrm{~Hz}$, so that 100 filters have been used for frequency domain filtration. The amplitude has been smoothed by running mean process with time duration 0.1 seconds. In Figure 7(a), the most strong energy with frequency between 2.0 to $4.0 \mathrm{~Hz}$ between time 6.0 to 10.0 seconds can be clearly identified. This area corresponds to the large amplitude and low frequency section of the accelerogram from 6.0 to 10.0 seconds. Very low frequency component between 1.0 to $2.0 \mathrm{~Hz}$ after 14.0 seconds is also clearly seen. The high frequency component can not be well separated. Big energy around $8 \mathrm{~Hz}$ at 7.0 to 8.0 seconds, between 12.0 to $14.0 \mathrm{~Hz}$ at 4.0 to 8.0 seconds can be observed. Viewing in the time domain, the early time of the accelerogram is mainly of high frequency component between 12.0 to $16.0 \mathrm{~Hz}$. The frequency decreases with time. Between 10.0 and 11.0 seconds, an impulse with wide frequency range is obvious. In general, the result does not show a clear pattern of the temporal frequency variation. As poor time resolution for low frequency range and poor frequency resolution for high frequency range, the signal are averaged in time domain at low frequency and in the frequency domain at high frequency. It is not so easy to identify and locate the frequency component within a wide frequency range. As a good resolution of frequency at low frequency and good time resolution at high frequency is suitable for display the dispersed seismic wave, the multiple filter technique is very successful in analyzing dispersed seismic wave.

For running spectrum analysis, a Bartlett moving time window of 1.27 seconds was applied to the accelerogram, so that the number of time window applied between 0.0 to 20.0 seconds was 100 . In order to obtain values for the beginning in time domain, several zeros were added to the beginning of accelerogram. The window moves in a time lag 0.2 seconds. The results were smoothed in the time domain by running mean process with a time duration of 0.6 seconds.

The results by MEM for this accelerogram is shown in Figure 7(b). The frequency interval is $0.1575 \mathrm{~Hz}$. The high energy is well located around frequency 2.0 to $4.0 \mathrm{~Hz}$ between 5.0 to 11.0 seconds, around $8.0 \mathrm{~Hz}$ at 6.0 to 7.0 seconds, around $14.0 \mathrm{~Hz}$ at about 3.0 and 6.0 seconds, around $7.0 \mathrm{~Hz}$ at about 12.0 seconds, and low frequency component around $2.0 \mathrm{~Hz}$ at 15.0 seconds. The beginning part of the accelerogram is of frequency around $14.0 \mathrm{~Hz}$, it lasts until around 4.0 seconds, then another wave type of nearly the same frequency arrives at about 4.5 seconds, it lasts until 6.0 seconds. From about 5.0 seconds, a low frequency wave arrives which lasts until about 9.0 seconds. Impulses of frequency around $8.0 \mathrm{~Hz}$ appears around 6.0 seconds and 12.0 seconds. An impulse of wide frequency range is observed around 
10.0 seconds. Comparing with the multiple filter analysis, the MEM result gives a very clear and detailed pattern of the time-frequency distribution. The frequency components are well separated and located in time domain. It is very clear to identify the appearing and evolution of different wave types. Since MEM analysis can achieve good frequency resolution for short time length, the time resolution is also improved. The selection of time window length is also arbitrary, the most suitable time window for both good time and frequency resolution can be found through test.

Figure $7(\mathrm{c})$ is the result of running spectrum by FFT. The frequency and time locations of the strong energy can also be clearly identified. The time resolution is very good and the starting and ending time of different wave types can be well observed. But the detailed frequency variation can not be separated. Comparing with the multiple filter analysis, it gives better time resolution for all frequency range. Its frequency resolution is worse than the MEM analysis. For running spectrum estimation by FFT, since the selection of time window is limited to $2^{n}$ data, the number of time window length can be used is limited to a few and varies greatly between each other. The frequency resolution is then also restricted. In this analysis, several time windows have been applied, and only the present result gives the best time and frequency resolution.

Comparing between the results of three methods, the running spectrum technique by MEM gives the best resolutions in both time and frequency domain. Because the selection of time window length for MEM is arbitrary, the appropriate window can be found through several calculations. The frequency resolution is not directly related to the time window length. The frequency resolution can also be selected by setting the appropriate frequency interval. The good frequency and time resolution can be achieved over wide frequency range for time series of not only long but also short duration. For running spectrum analysis by FFT, selection of time window is limited and the frequency resolution is directly related to the window length. If the time window is selected, the frequency resolution is also selected at the same time. For a time series of long duration, it is not easy to identify the detailed frequency variation over a wide range of frequency. For a short section of a seismogram containing simple frequency content, it can be used to achieve good frequency and time resolution. The multiple filter technique is especially good for analyzing a short section of seismogram of mainly dispersed wave.

For analyzing this accelerogram, the CPU time used by multiple filter technique is 48.7 seconds, running spectrum analysis by MEM is 11.0 seconds, by Fourier transform is 2.2 seconds on a SUN SPARC 5 work station. It is more time consuming to carry out multiple filter analysis than running spectrum analysis for the same time signal. The multiple filter technique also produces very big data matrix compared with the other two methods.

\section{Conclusions}

The three methods for time-frequency analysis are compared between each other. For all of them, the time and frequency resolution is restricted. For multiple filter technique, the relative bandwidth is a controlling parameter, it should be chose according to the interested frequency range and time range. Multiple filter analysis is proved to be a very successful tool to analyze dispersed seismic wave. It is suitable for a short section of seismic signal containing mainly dispersed waves. The running spectrum analysis by MEM can achieve 
both good time and frequency resolutions, and can be applied for signals of both long and short duration. The running spectrum analysis by Fourier transform has poorer frequency resolution for the same time resolution comparing with MEM and seems not very good for long time series.

Graphic display technique is important for visualizing the results. What kind of display technique should be used is determined by the characteristics of the outputted data.

One should select the method prior to performing time-frequency analysis. In applications, which method should be adopted is closely related to the purpose of analyzing and the property of the time signal.

\section{Acknowledgments}

The accelerogram used in this study is from the Kyoshin Net (K-NET) strong motion database of Japan on Internet.

\section{References}

AVIJIT, C., and DAVID, O. (1995): Frequency-time decomposition of seismic data using wavelet-based methods. Geophysics, 60, (6), 1906-1916.

CHUNG, J., and YEONG, T. (1997): Shallow crustal structure from short-period Rayleigh-wave dispersion data in southwestern Taiwan. Bull. Seism. Soc. Am., 87, (2), 370-382.

DOO, J. J., and RICHARD J.C. (1991): A basic program to compute seismic surface-wave groupvelocity dispersion curves. Computers \& Geosciences, 17, (6), 777-799.

DZIEWONSKI, A., BLOCH, S., and LANDISMAN, M. (1969): A technique for the analysis of transient seismic signals. Bull. Seism. Soc. Am., 59, (1), 427-444.

FuRUMURA, M. (1994): Research on the response of Tokachi plain to long period seismic motion (in Japanese), Ph. D. thesis, Depart. Earth \& Planet. Sci., Hokkaido University.

Hino, M. (1977): Spectrum analysis (in Japanese), Asakura shoten, Tokyo.

KoBAYASHI, M., and SAKAMOTO M. (1997): Wavelet-based time-frequency analysis : speech signals, Workshop on Seismology and Wavelets, University of Tokyo, Tokyo.

MEYER, Y. (1991): Wavelets and applications, Springer-Verlag, Berlin.

PAPOUlis, A. (1977): Signal analysis, 273p., McGraw-Hill, New York.

William, H. P., SaUl, A. T., William, T. V., and BRIAN, P. F. (1992): Numerical recipes in FORTRAN, Cambridge University Press, New York.

YokoI, T. (1996): A later phase detected by the tripartite observation at Sumiyoshi, Kobe, Report of comprehensive study of strong ground motion prediction, DPRI, Kyoto University, 147-158.

ZHENG, Z.Z., and ZHANG, S. F. (1993): Theory and its applications of transient spectrum estimation (in Chinese), Seismological Press, Beijing. 